\title{
Research on Three-Dimensional visualization of Data Center Scene
}

\author{
Ma Jianwei ${ }^{a}$, Wan Huijiang ${ }^{b}$, Liang Ling ${ }^{c}$ Jiang Zaineng ${ }^{d}$ \\ Guizhou Power Grid Co., Ltd., Power Dispatching Control Center, Guiyang, Guizhou 550002 China \\ a'425054771@qq.com, b408438504@qq.com, '361550717@qq.com, d991980142@qq.com
}

Keywords: Electric power system; Control center; Three-dimensional visualization; Spatial interpolation algorithm

\begin{abstract}
In view of the node type and line type data in the control center of electric power system, the visualized expression based on wiring diagram of power grid, from three-dimensional curved surface diagram, three-dimensional piping diagram, three-dimensional arrow diagram to three-dimensional mapping diagram, was designed respectively, as well as the commonly used three-dimensional visual theme. The spatial interpolation algorithm in the curved surface diagram was improved and the application of color table mapping was discussed.
\end{abstract}

\section{Introduction}

With the continuous expansion of the scale of the power grid, the electric power system is becoming increasingly complicated, and the type and number of data produced in the system running are greatly increased. In the wiring diagram of power grid, though the traditional data expression annotated by figure has the advantages of fast display and accurate data, it is not intuitive enough. It is difficult for this expression to identify key information from a large number of data, and to meet the needs of real-time monitoring with large power grid. The three-dimensional visualization technology makes full use of third dimensions in the space. Compared with traditional two-dimensional display, it can express a large number of information, and express a large number of data in the power grid in a more efficient and intuitive manner, which helps the dispatcher to quickly understand the problem and its severity in the current system, allowing the dispatcher to grasp the overall running of power grid. All the advantages have caused wide attention of scholars and even the whole industry, and have become one of the important characteristics of the new generation of energy management system (EMS). The research on visualization of power system is based on the power network. In the electric power system, the three-dimensional visualization technology based on power grid is further researched practically, which has better solved the problems of real-time, system integration and cross platform. As the centralized display platform of dispatching information, the research on three-dimensional dynamic intelligent display technology of real-time information in power grid and the application project can help to solve the actual problems, for example, the communication of data system between each business system, as well as the cross matching, cannot be achieved, and the dispatching screen cannot be fully used to make the real-time dynamic display of the large number of information. To truly realize the intelligently centralized display of real-time information, to pursue a more beautiful and dynamic in the practical basis, to fully consider the humanistic care, to fully optimize the design from the view of human engineering, and to eliminate the visual fatigue, aesthetic fatigue on the dispatching screen, then the dispatchers can be more efficient in their daily work, and the research can be used throughout the whole country.

\section{Three-dimensional visualization of data center scene}

\subsection{Expression}

There are many monitoring data in the real-time operation of electric power system, therefore they need to be classified, and the appropriate expression should be chose according to the 
characteristics of different types, then the visualized themes can be designed. The visualized themes commonly used in EMS are shown in Table 1.

Table 1 Visual theme design in common use in EMS

\begin{tabular}{|c|c|c|}
\hline Themes & Types & Visualized expression \\
\hline \multirow[t]{2}{*}{ The voltage type } & bus voltage in power station & $\begin{array}{l}\text { three-dimensional histogram, } \\
\text { curved surface chart }\end{array}$ \\
\hline & the amount of voltage & three-dimensional histogram \\
\hline \multirow{3}{*}{ Non-power type } & non-power margin of rotating equipment & $\begin{array}{l}\text { three-dimensional histogram, } \\
\text { pie chart }\end{array}$ \\
\hline & non-power margin of fixed equipment & $\begin{array}{l}\text { three-dimensional histogram, } \\
\text { pie chart }\end{array}$ \\
\hline & the total of non-power margin & $\begin{array}{l}\text { three-dimensional histogram, } \\
\text { pie chart }\end{array}$ \\
\hline \multirow{3}{*}{ Power type } & the load or output of power station & $\begin{array}{l}\text { three-dimensional histogram, } \\
\text { pie chart }\end{array}$ \\
\hline & the power flow of line & $\begin{array}{l}\text { three-dimensional mapping } \\
\text { chart, arrow chart }\end{array}$ \\
\hline & the power loss of line & three-dimensional mapping chart \\
\hline \multirow{2}{*}{ Stability index type } & $\begin{array}{l}\text { the voltage stability margin index(the } \\
\text { collapse distance of voltage) }\end{array}$ & $\begin{array}{c}\text { three-dimensional histogram, pie } \\
\text { chart }\end{array}$ \\
\hline & $\begin{array}{l}\text { the ATC (available transfer capacity) index } \\
\text { of crucial section }\end{array}$ & $\begin{array}{c}\text { three-dimensional histogram, pie } \\
\text { chart }\end{array}$ \\
\hline
\end{tabular}

\subsection{Basic algorithm}

Before users enter the virtual scene of data center by using operating platform, a large amount of resources including scenes and models need to be loaded, often it needs time to wait, and if the waiting time is too long, user's experience is bound to be affected, then dynamic load need to be used. Thus Unity 3D engine introduced AssetBundle. Provided by Unity3D engine, AssetBundle is a kind of file format that can store any kind of Unity3D, identify and store resources. Developers can pack and compress the resources that may be used to the server, then load them according to actual need, and load it dynamically by loading modules. If a resource is cited by multiple resources, then the dependency relationship between AssetBundle files can be established to reduce the final AssetBundle file.

\subsection{Spatial interpolation algorithm}

1. Spatial interpolation algorithm and its improvement

In three-dimensional curved surface mapping, the key step is to select the appropriate spatial interpolation algorithm and extend the data defined on the discrete point to the whole plane. As the example shown in Fig 1, we have defined the 4 point data of V1-V4 on the plane, $\mathrm{P}$ is any point in the plane, and the task of the spatial interpolation algorithm is to calculate the virtual value $v_{p}$ of $p$ point.

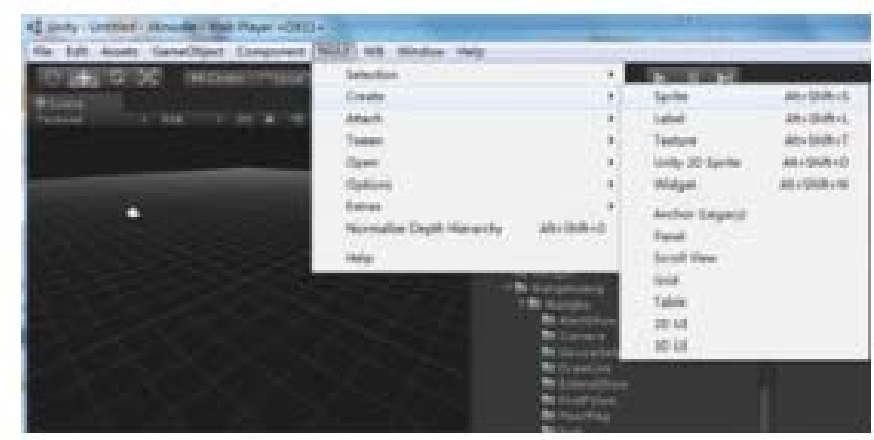

Fig.1 NGUT plug-in 


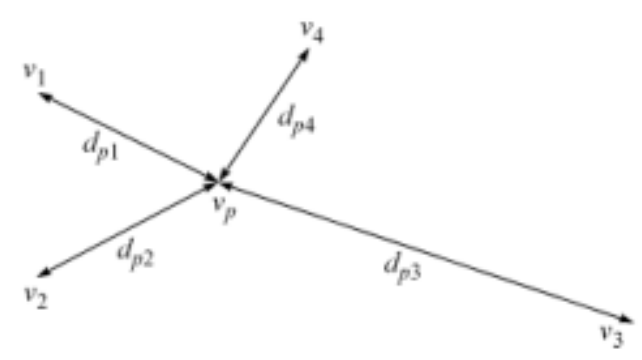

Fig.2 Example of spatial interpolation algorithm

The most common spatial interpolation algorithm is distance squared inverse algorithm, its formula is:

$$
v_{p}=\frac{\sum_{i=1}^{N} \frac{v i}{d_{p i}^{2}}}{\sum_{i=1}^{N} \frac{1}{d_{p i}^{2}}}
$$

In the formula: $\mathrm{N}$ is the number of known data points; $\mathrm{d}_{\mathrm{pi}}$ is the distance from the data point $\mathrm{i}$ to p. In practice, it is generally necessary to interpolate each point on the two-dimensional grid with large amount of calculation. The spatial interpolation algorithm is optimized by 2 points. First, in the formula (1), only the influence of the nearer data points from the point $\mathrm{p}$ is considered, that is,

$$
v_{p}=\frac{\sum_{\substack{i=1 \\ d_{p i}<d_{\text {inf }}}}^{N} \frac{v i}{d_{p i}^{2}}}{\sum_{\substack{i=1 \\ d_{p i}<d_{\text {inf }}}}^{N} \frac{1}{d_{p i}^{2}}}
$$

In the formula: dinf is a preset influence distance, and each data point only affects the area of a distance less than dinf. Secondly, the number of calculation times of the distance is reduced to the original $1 / 8$ by using the symmetry of the square grid in integer coordinates. But after the above improvements, the algorithm still has shortcomings: being unable to calculate the point that need to be interpolated outside all the influence area of data points, and the result is not continuous at the boundary of the influence region, and the interpolation point is always the same as the data point in the area affected by one data point. The above deficiencies affect the visual effect of the interpolated graphics. Therefore a new fast interpolation algorithm is proposed in this paper, its formula is:

$$
v_{p}=\frac{c v \infty+\sum_{\substack{i=1 \\ d_{p i}<d_{\text {inf }}}}^{N}\left\{\frac{1}{d_{p i}^{2}}-\frac{1}{d_{\text {inf }}^{2}}\right\}}{c+\sum_{\substack{i=1 \\ d_{p i}<d_{\text {inf }}}}^{N}\left\{\frac{1}{d_{p i}^{2}}-\frac{1}{d_{\text {inf }}^{2}}\right\}}
$$

In the formula: $\mathrm{C}$ is a smooth constant that regulates the smoothness of the interpolation result in the transition at the boundary of the region. In theory, the known $\mathrm{N}$ data points is interpolated in the M? M grid, and the calculation of multiplication and division of the original algorithm (equation (1)) is proportional to $\mathrm{NM}^{2}$, and when $\mathrm{d}_{\text {inf }}$ is set to $\mathrm{S} / \mathrm{N}$ according to the experience( $\mathrm{S}$ is the interpolation area), the improved calculation of multiplication and division is only proportional to $\mathrm{M}^{2}$, the calculation is greatly reduced. The algorithm is calculated at a rapid speed, and through introducing the virtual average $(\mathrm{v} \infty)$ and weight correction $\left(1 / \mathrm{d}^{2}\right.$ inf $)$, the disadvantage of the algorithm thus used is eliminated: outside the influence area of all the data points, the interpolation results $\mathrm{vp}=\mathrm{v} \infty$, at the 
boundary of the influence area of the i point, the weight coefficient of vi $1 / \mathrm{d}^{2}{ }_{\mathrm{pi}}-1 / \mathrm{d}^{2}{ }_{\text {inf }}=0$, ensure the continuity of the calculation result; in the influence area of only one data point, the interpolation result is the weighted average of the numerical and $\mathrm{v} \infty$, the change continuous. Besides, the location of data points is approximated to grid points under the rectangular grid of floating point coordinate, and the distance calculation in the whole influence area is completed on the 1/4 circle with the use of symmetry, so that the amount of calculation can be reduced by 3/4. Through the improvement of the interpolation algorithm, the real time and practicability of the three-dimensional curved surface chart have been greatly improved.

\section{The effect of operation platform in data center}

The visualized scene management method is used in the visulaized scene management of three-dimensional of data center, the running systems are as follows: CPU: Intel(R) Xeon E5-2630 2.60GHz; RAM:64GB; GPU: NVIDIA Quadro K4000; the operating system is Windows7 64bit, the scene is shown in Fig.3:

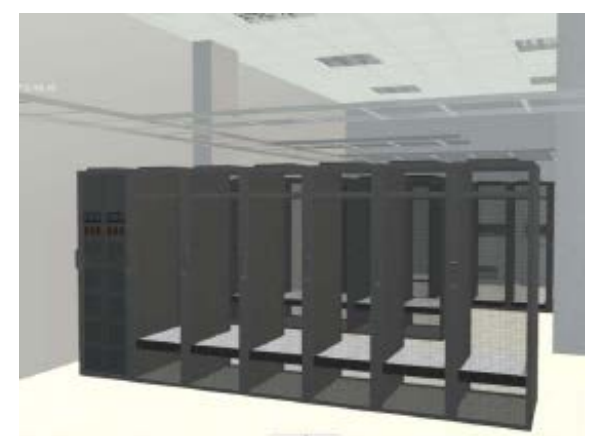

Fig.3 The three-dimensional visualized scene of data center

Part of the equipment in the three-dimensional scene of data center is listed when display condition is switching, so as to illustrate the real-time characteristic of scene management method acted on the control of display condition:

Table 2 The comparison of the display conditions when they are switching

\begin{tabular}{|c|c|c|c|}
\hline method & frame & air-conditioner & UPS \\
\hline text method & $49 \mathrm{~ms}$ & $36 \mathrm{~ms}$ & $27 \mathrm{~ms}$ \\
\hline octree method & $131 \mathrm{~ms}$ & $83 \mathrm{~ms}$ & $64 \mathrm{~ms}$ \\
\hline cell-portal method & $96 \mathrm{~ms}$ & $75 \mathrm{~ms}$ & $50 \mathrm{~ms}$ \\
\hline
\end{tabular}

The scene management method ensures the geometric completeness of objects, the targeted form is used to show scene object in the scene management model. When the controlling object display condition, there is no need to update the data of multiple nodes in scene management structure. Therefore when display condition of the equipment is switching, the above method thus mentioned is better than octree method, cell-portal method, the real-time control of the display condition of object has been achieved.

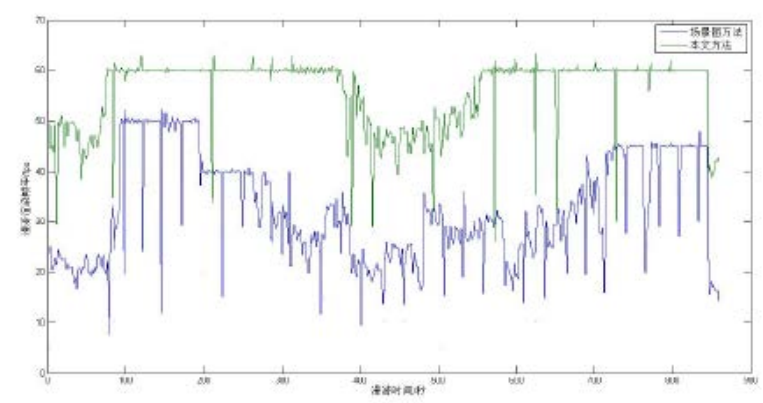

Fig.4 Diagram four the comparison of the roaming scene frame frequency between scene method and text method 
By using the application of three-dimensional visualization, the operation system can dynamically load resources, certify identification, make integrated enquiry, monitor system, manage daily record, prompt alarm information, help attendants to maintain and make real-time supervision of the operating condition of data center, and to fulfill daily management of the operation platform, the effect is shown in Fig.5.

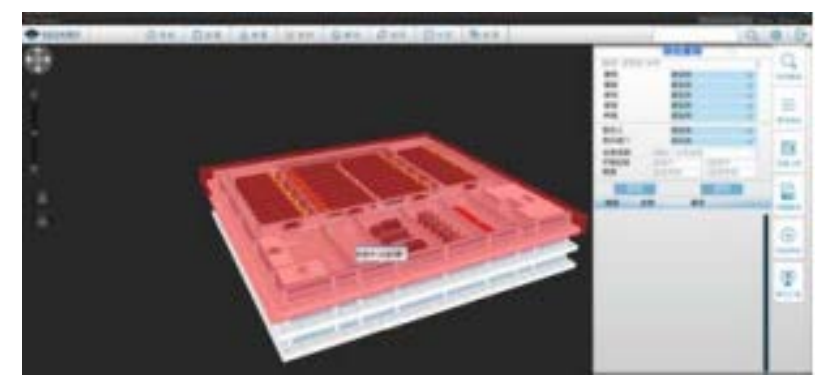

Fig.5 The effect graph of three-dimensional operation platform

\section{Conclusions}

In accordance with the trend and requirements of establishing unified, strong and intelligent power grid, with capital construction of informationization as its core. By using the management system of three-dimensional visualized construction, the all-around, deep-going control and monitor of quality, technology, security, economy can be achieved, the common operation, process and management method be innovated, the capital construction and informationization can be promoted, which is of significance in improving power grid engineer and management. However, research on the system is in its initial stage, shortcomings are inevitable, for example, the standard of data form is not comprehensive, the long and thorough research should be made in all aspects to make further improvements and optimization.

\section{References}

[1] Zhu Chuanlin, Wang Xueliang, Fan Hongfei, Chaijian, Yurong, Wang Zihe. Design and Implementation of Three-dimensional Visualized Statistical Analysis System for Lightning Data [J]. Meteorological Science and Technology, 2017,01:58-62+66.

[2] Wang Lei, Lian Dongben, Wang Junlin. The Three-dimensional Operation Platform of Data Center[J]. Application of Computer System, 2017,03:260-263.

[3] Wang Lei, Lian Dongben, Wang Junlin. The Design of Editor in the Three-dimensional Visualized Scene of Data Center[J]. Computer System Application, 2017,05:253-256.

[4] Mai Xiaoming, Chen Chi, Peng Xiangyang, Zhang Boyu, Wang Ke, Yang Bisheng, Wang Rui. Three-dimensional Visualization Technology and System Design of Transmission Line Corridor[J]. China Electric Power, 2015,02:98-103.

[5] Luo Junsong, Deng Fei, Lu Hanyu, Luo Junkai. Research of the Three-dimensional Visualization Technology based on Qt [J]. Computer Measurement and Control,2013,03:797-799.

[6] Wang Jiaojiao. Three-dimensional Visualized Research Progress of Vector Data[J]. Guangdong Agricultural Sciences, 2013,08:183-188. 